\title{
Stability Analysis of a Dynamical Model Representing Gene Regulatory Networks *
}

\author{
Mehmet Eren Ahsen* Hitay Özbay** \\ Silviu Iulian Niculescu ${ }^{* * *}$ \\ * Department of Bioengineering, University of Texas at Dallas, \\ Richardson, TX 75080-3021, USA (e-mail: ahsen@utdallas.edu). \\ ** Department of Electrical and Electronics Engineering, Bilkent \\ University,06800 Ankara,Turkey (e-mail: hitay@ee.bilkent.edu.tr) \\ *** Laboratoire des Signaux et Systèmes (LSS), CNRS-SUPELEC, \\ 3 rue Joliot Curie, 91192 Gif-sur-Yvette France (e-mail: \\ Silviu.Niculescu@lss.supelec.fr)
}

\begin{abstract}
In this paper we perform stability analysis of a class of cyclic biological processes involving time delayed feedback. More precisely, we analyze the genetic regulatory network having nonlinearities with negative Schwarzian derivatives. We derive a set of conditions implying global stability of the genetic regulatory network under positive feedback. As a special case, we also consider homogenous genetic regulatory networks and obtain an appropriate stability condition which depends only on the parameters of the nonlinearity function.
\end{abstract}

Keywords: Gene Regulatory Networks, Schwarzian Derivatives, Asymptotic Stability, Hill Functions, Nonlinear Time Delay Networks.

\section{INTRODUCTION}

In this work, we will be concerned with the asymptotic stability of a class of biological systems, the so-called gene regulatory networks with delayed feedback (see, e.g. Smolen et al. (2000a,b)). Basically, a gene regulatory network can be described as the interaction of DNA segments with themselves and with other biological structures such as the enzymes in the cell. Therefore, it can be thought as an indicator of the genes transcription rates into mRNA, which is used to deliver the coding information required for the protein synthesis, see e.g. Levine and Davidson (2005). The model proposed in Chen and Aihara (2002) consists of a set of differential equations in the following form:

$$
\left\{\begin{aligned}
\dot{p}_{1}(t) & =-k_{p 1} p_{1}(t)+f_{p 1}\left(g_{m}\left(t-\tau_{g m}\right)\right) \\
\dot{g}_{1}(t) & =-k_{g 1} g_{1}(t)+f_{g 1}\left(p_{1}\left(t-\tau_{p 1}\right)\right) \\
& \vdots \\
\dot{p}_{m}(t) & =-k_{p m} p_{m}(t)+f_{p m}\left(g_{m-1}\left(t-\tau_{g_{m-1}}\right)\right) \\
\dot{g}_{m}(t) & =-k_{g 1} g_{m}(t)+f_{g m}\left(p_{m}\left(t-\tau_{p m}\right)\right),
\end{aligned}\right.
$$

where $p_{i}$ and $g_{i}$ represent the protein and mRNA concentrations respectively. Models similar to (1) are frequently encountered in the modeling of biological processes such as mitogen-activated protein cascades and circadian rhythm generator Goldbeter (1996), Townley et al. (1999) and Sontag (2002). For instance, in Chen and Aihara (2002), a simplified version of the system (1) is analyzed and a local stability result is given. An explicit computation of the allowable upper bounds on the delay value can be found in Morarescu and Niculescu (2008).

\footnotetext{
* This work is supported in part by the French-Turkish PIA Bosphorus (TUBITAK Grant No. 109E127 and EGIDE Project No. $22974 \mathrm{WJ})$, and by DPT-HAMIT project.
}

The system (1) under single time-delay and negative feedback has been studied and discussed in Enciso (2006), where an easy condition for asymptotic stability has been obtained. By using a Hopf bifurcation approach Enciso (2006) showed the existence of oscillations in some cases. He also used the arguments used in Liz et al. (2003), Angeli and Sontag (2004) to embed the system (1) to a discrete time system. In the present work, we will analyze the gene regulatory network under positive feedback using some of the results of Ahsen (2011). We will assume that the functions $f_{p i}$ and $f_{g i}$ are nonlinear and have negative Schwarzian derivatives. As a subcase of the positive feedback, we will consider the homogenous gene regulatory network and obtain a sufficient condition for asymptotic stability of the system which depends only on the parameters of the nonlinearity function.

The remaining parts of the paper are organized as follows: In the next section we formulate the problem studied and give some preliminary results. In Section 3 the main results are stated. Illustrative examples are given in Section 4 , and concluding remarks are made in the last section.

\section{NOTATION, PRELIMINARIES AND PROBLEM FORMULATION}

In this section, we present some basic definitions and notations that are frequently used in the paper. For the analysis of system considered here we will use properties of Schwarzian derivatives, that are commonly employed in analysis of these types of cyclic nonlinear feedback systems, see e.g. Müller et al. (2006). Let a function $f$ be defined from $\mathbb{R}_{+}$to $\mathbb{R}_{+}$. Suppose it is at least three times continuously differentiable. Then, the Schwarzian 
derivative of the function $f$, see Sedeghat (2003), denoted as $S f(x)$, is given by the following:

$$
S f(x)= \begin{cases}-\infty & \text { if } f^{\prime}(x)=0 \\ \frac{f^{\prime \prime \prime}(x)}{f^{\prime}(x)}-\frac{3}{2}\left(\frac{f^{\prime \prime}(x)}{f^{\prime}(x)}\right)^{2} & \text { if } f^{\prime}(x) \neq 0\end{cases}
$$

We use the notation $f^{m}$ to denote the function obtained by $m$ compositions of $f$. We say that $x$ is a fixed point of $f$ if $f(x)=x$.

In the sequel, we analyze the following simplified system which is equivalent to (1), where we have a single delay in the feedback channel:

$$
\left\{\begin{aligned}
\dot{x}_{1}(t) & =-\lambda_{1} x_{1}(t)+g_{1}\left(x_{2}(t)\right) \\
\dot{x}_{2}(t) & =-\lambda_{2} x_{2}(t)+g_{2}\left(x_{3}(t)\right) \\
& \vdots \\
\dot{x}_{n}(t) & =-\lambda_{n} x_{n}(t)+g_{n}\left(x_{1}(t-\tau)\right) .
\end{aligned}\right.
$$

Note the following relation between $\tau$ and $\tau_{g i}, \tau_{p i}$ :

$$
\tau=\sum_{i=1}^{m}\left(\tau_{p i}+\tau_{g i}\right) .
$$

In Section 3, we present conditions for the asymptotic stability and existence of oscillations regarding the nonlinear time delayed feedback system (2) under the following simplifying assumptions.

Assumption 1 For all $i=1,2, \ldots, n$, we have $\lambda_{i}>0$. Assumption 2 For all $i=1,2, \ldots, n$, the nonlinearity functions $g_{i}$ satisfy:

(i) $g_{i}(x)$ is a bounded function defined on $\mathbb{R}_{+}$;

(ii) we have either

$$
g_{i}^{\prime}(x)<0 \quad \text { or } \quad g_{i}^{\prime}(x)>0 \quad \forall x \in(0, \infty) .
$$

Assumption 2 means that each $g_{i}$ is a monotone function and takes positive values. The nonlinearity functions have $\mathbb{R}_{+}$as their domain since their domain represents biological variables which take positive values. Also note that $g_{i}^{\prime}(0)=0$ is allowed, since it does not violate the monotonicity of $g_{i}$. We will now define a new function $g$ in the following way:

$$
g=\left(\frac{1}{\lambda_{1}} g_{1}\right) \circ\left(\frac{1}{\lambda_{2}} g_{2}\right) \circ \ldots \circ\left(\frac{1}{\lambda_{n}} g_{n}\right) .
$$

Definition 1 . We say that the gene regulatory network is under positive feedback if

$$
g^{\prime}(x)>0 \quad \forall x \in(0, \infty) .
$$

Conversely, the gene regulatory network is said to be under negative feedback if the above inequality is reversed.

In this work, we will only be concerned with the positive feedback case. For the negative feedback case we refer to Ahsen (2011).

Throughout the paper, we will make use of the following result.

Theorem 1. (Smith (2008)). Consider the system (2) under positive feedback. Any solution (2) with any nonnegative initial condition converges to one of its equilibrium points.

The above result is very important in the sense that the solution does not diverge or show oscillatory behavior.
However, the system may have a number of equilibrium points, so which one is the attractor for a given initial condition is not specified. Moreover, it is important to identify the conditions under which we have a single equilibrium point and multiple equilibrium points. In this paper we will deal with these issues.

First obvious consequence of Theorem 1 is that when we have single equilibrium point, we have global stability (all non-negative initial conditions are brought to the equilibrium point). In the following Corollary a condition for single equilibrium is also given.

Corollary 2. Consider system (2) under positive feedback. If the function $g$ defined in (5) has a unique fixed point, then the system (2) has a unique equilibrium point $x_{e q}$ and any solution of the system with a non-negative initial condition will converge to its unique equilibrium point $x_{e q}$.

Proof. If $g$ has a unique fixed point, then it is shown in Ahsen (2011) that the system has a unique equilibrium point. The global convergence result follows directly from Theorem 1.

\section{ANALYSIS OF THE CYCLIC NETWORK UNDER POSITIVE FEEDBACK}

In the sequel, we will analyze system (2) subject to positive feedback. We assume that the nonlinearity functions have negative Schwarzian derivatives and Assumptions 1 and 2 are satisfied.

Proposition 1. Consider the system (2) under positive feedback and assume that $g$ defined in (5) has negative Schwarzian derivative. Then, the following results hold:

(i) The function $g$ has at most three fixed points.

(ii) If

$$
g^{\prime}(x)<1 \quad \forall x \geq 0,
$$

then $g$ has a unique fixed point. In this case, the system defined by (2) has a unique equilibrium point $x_{e q}$ which is globally attracting.

(iii) If $g^{\prime}(0)>1$ then $g$ has a unique positive fixed point.

Proof. See Ahsen (2011).

Therefore, if $g$ satisfies conditions (ii) or (iii) of Proposition 1 , then the unique equilibrium point of the system (2) is globally attractive.

\subsection{Homogenous Gene Regulatory Network under Positive Feedback}

In this section we deal with homogenous gene regulatory network under positive feedback. Consider system (2) under positive feedback with

$$
g_{i}(x)=f(x), \quad \lambda_{i}=1 \quad \forall i=1,2, \ldots, n .
$$

Notice that we did not assume any special form for $f$ yet. We start our analysis with the following Lemma:

Lemma 3. Let $k(x): R_{+} \rightarrow I \subseteq \mathbb{R}_{+}$be a three times continuously differentiable function satisfying

$$
k^{\prime}(x)>0 \quad \forall x \in(0, \infty) .
$$

Let $h$ be defined on $\mathbb{R}_{+}$as

$$
h(x)=k^{m}(x) .
$$

Then, any fixed point of $h$ is a fixed point of $k$. 
Proof. Suppose that $h(0)=0$ and $k(0)>0$ then we have

$$
h(0)=k^{n}(0)>\ldots>k(k(0))>k(0)>0
$$

which is contradiction. Therefore, $k(0)=0$ and 0 is a fixed point of the function $k$. Let $x>0$ be a fixed point of the function $h$ and suppose $k(x) \neq x$. Then we have either

$$
x<k(x) \quad \text { or } \quad k(x)<x .
$$

If $x<k(x)$, then since $k$ is a strictly increasing function we have

$$
h(x)=k^{n}(x)>\ldots>k(x)>x,
$$

which gives us a contradiction. Similarly, if we have $k(x)<$ $x$ then

$$
h(x)=k^{n}(x)<\ldots<k(x)<x
$$

which is again a contradiction. Therefore, we should have $k(x)=x$. Also, it is easy to see that any fixed point $x$ of $k$ is a fixed point of $h$. Thus we conclude that the functions $k$ and $h$ have the same fixed points.

Remark 4. The homogenous system is under positive feedback either if

(i) $f^{\prime}(x)>0$ for all $x \in(0, \infty)$ or

(ii) $f^{\prime}(x)<0$ for all $x \in(0, \infty)$ and $n=2 m$ for some positive integer $m$.

We will first deal with the case (ii) of Remark 4. From linear algebra, we know that every positive number has a unique prime decomposition. We also know that $n$ is an even integer. Then, we have either

(i) $n=2^{l}$ for some positive integer $l$ or

(ii) $n=2^{l_{1}} p_{2}^{l_{2}} \ldots p_{n}^{l_{n}}$, where $p_{2}, p_{3}, \ldots, p_{n}$ are distinct odd primes and $l_{i}>0$.

We have the following Lemma regarding case (ii) of Remark 4:

Lemma 5. Consider the homogenous gene regulatory network (2) under positive feedback with

$$
f^{\prime}(x)<0 .
$$

Moreover, suppose that $f$ has negative Schwarzian derivative. Then, $f$ has a unique fixed point, say $x_{0}>0$, and one of the following holds:

(i) We have $n=2^{l}$. In this case

$$
g(x)=f^{n}(x)
$$

has the unique fixed point $x_{0}$ provided that

$$
\left|f^{\prime}\left(x_{0}\right)\right|<1 \text {. }
$$

If $\left|f^{\prime}\left(x_{0}\right)\right|>1$, then $g$ has exactly three equilibrium points. (ii) When $n=2^{l_{1}} p_{2}^{l_{2}} \ldots . p_{n}^{l_{n}}$, we define $h$ as

$$
h(x)=f^{(P)}(x),
$$

where $P=\prod_{i=2}^{n} p_{i}^{l_{i}}$. In this case $h$ has a unique fixed point $x_{0}$ which is also the unique fixed point of $f$. If

$$
\left|f^{\prime}\left(x_{0}\right)\right|<1
$$

then we have $\left|h^{\prime}\left(x_{0}\right)\right|<1$ and $g$ defined in (6) has the unique fixed point $x_{0}$. If we have

$$
\left|f^{\prime}\left(x_{0}\right)\right|>1 \text {, }
$$

then $\left|h^{\prime}\left(x_{0}\right)\right|>1$ and $g$ defined in (6) has exactly three equilibrium points.

Proof. Firstly, since $f$ is monotonically decreasing we know that it has a unique fixed point $x_{0}$. Suppose $n=2^{l}$ and let

$$
g(x)=f^{n}(x) .
$$

Now, let $h_{1}(x)=f^{2^{l-1}}(x)$, then we have

$$
g(x)=h_{1}\left(h_{1}(x)\right) \quad \text { and } \quad h_{1}^{\prime}(x)>0 \quad \forall x \in(0, \infty) .
$$

From Lemma 3 with $m=2$, we conclude that any fixed point $x$ of $g$ is a fixed point of the function $h_{1}$. Let $h_{2}(x)=f^{2^{k-2}}(x)$, then we have

$$
h_{1}(x)=h_{2}\left(h_{2}(x)\right)
$$

and again from Lemma 3 we conclude that any fixed point of $h_{1}$ is a fixed point of $h_{2}$. Since $n=2^{l}$ we know that $g$ has as many fixed points as $h_{l-1}$ which is defined as

If we have

$$
h_{l-1}(x)=f(f(x)) \text {. }
$$

$$
\left|f^{\prime}\left(x_{0}\right)\right|<1
$$

at the unique equilibrium point $x_{0}$ of $f$, we conclude that $h_{l-1}$ has a unique equilibrium point. Therefore, from Lemma 3 we deduce that $g$ has a unique fixed point. Lemma 3 also implies that if

$$
\left|f^{\prime}\left(x_{0}\right)\right|>1
$$

then the function $h_{l-1}(x)$ has exactly three fixed points. Therefore, from Lemma 3 the function $g(x)$ has three fixed points.

Now for the second part, consider $n=2^{l_{1}} p_{2}^{l_{2}} \ldots . p_{n}^{l_{n}}$ and let

$$
P=p_{2}^{l_{2}} \ldots p_{n}^{l_{n}} \text {. }
$$

and

$$
h(x)=f^{P}(x) .
$$

Since $P$ is an odd number, we have

$$
h^{\prime}(x)<0 \quad \forall x \in(0, \infty) .
$$

We also know that $h$ has negative Schwarzian derivative by the convolution property of Schwarzian derivatives, see Sedeghat (2003). Therefore, $h$ has a unique fixed point. Since $f$ is decreasing it has a unique fixed point $x_{0}$. Also note that

$$
h\left(x_{0}\right)=f^{P}\left(x_{0}\right)=x_{0},
$$

from which we conclude that the unique fixed point $x_{0}$ of $f$ is the unique fixed point of $h$. Also note that

$$
\left|h^{\prime}\left(x_{0}\right)\right|<1 \Leftrightarrow\left|f^{\prime}\left(x_{0}\right)\right|<1 .
$$

Similarly, we have

$$
\left|h^{\prime}\left(x_{0}\right)\right|>1 \Leftrightarrow\left|f^{\prime}\left(x_{0}\right)\right|>1 .
$$

Notice that

$$
g(x)=h^{2^{l_{1}}}(x) .
$$

Then the rest of the arguments are the same as the proof of the first part.

We will continue our analysis with case (i) of Remark 4 . We consider the homogenous gene regulatory network under positive feedback with $f$ satisfying

$$
f^{\prime}(x)>0 \quad \forall x \in(0, \infty)
$$

Lemma 6 . Consider the homogenous gene regulatory network (2) under positive feedback with the nonlinearity function $f$ satisfying (8). Then, the function

$$
g(x)=f^{n}(x)
$$

has as many fixed points as $f$. In particular, if $f$ has a unique fixed point, then system (2) has a unique equilibrium which is globally attractive.

Proof. Lemma 3 and Proposition 1 gives us the desired result. 
We are interested in the fixed points of the function $f$. If, further, $f$ has a negative Schwarzian derivative, we know that it has one, two or three fixed points. As an example, let us consider the following Hill type of functions and try to find some conditions regarding its fixed points. The type of functions we will consider is given by

$$
f(x)=\frac{a x^{m}}{b+x^{m}}+c, \quad a, b, c>0
$$

so we rule out zero as a fixed point by taking the constant $c$ strictly positive. Then $x>0$ is a fixed point of the function defined in (9) if $x$ is a root of the following polynomial:

$$
h(x)=x^{m+1}-(a+c) x^{m}+b x-b c .
$$

Some interesting cases regarding the function (10) may occur. Let us consider one such interesting example. Let $a=3.6, b=5, m=2$ and $c=0.4$, then we have

$$
h(x)=x^{m+1}-(a+c) x^{m}+b x-b c=(x-1)^{2}(x-2)
$$

which implies that the function $f$ has exactly two fixed points.

We will try to find a sufficient condition depending on the parameters $a, b, c$ and $m$ so that the function $f$ defined in (9) has a unique equilibrium point. First note that for arbitrary positive constants $a, b, c$ and $m$, we have

Therefore, if we have

$$
h(0)=-b c<0 \text {. }
$$

$$
h^{\prime}(x) \geq 0 \quad \forall x \in \mathbb{R}_{+},
$$

then $h$ can have at most one positive root so $f$ has a unique fixed point. For $m>1$, we have

$$
\begin{aligned}
h^{\prime}(x) & =(m+1) x^{m}-(m)(a+c) x^{m-1}+b \\
& =x^{m-1}((m+1) x-m(a+c))+b=h_{1}(x)+b .
\end{aligned}
$$

In order to guarantee (11), we should have

$$
h_{1}(x) \geq-b \quad \forall x \in \mathbb{R}_{+} .
$$

But $h_{1}$ takes its minimum at the point $y$ where

$$
h_{1}^{\prime}(y)=0 \text {. }
$$

As a result of (12), we get the following equations:

$$
\begin{aligned}
h_{1}^{\prime}(x) & =(m+1)(m) x^{m-1}-(m)(m-1)(a+c) x^{m-2} \\
& =x^{m-2}(m)(m+1)\left(x-\frac{m-1}{m+1}(a+c)\right) \\
& \Rightarrow h_{1}^{\prime}(y)=0 \Leftrightarrow y=\frac{m-1}{m+1}(a+c) \\
& \Rightarrow \min \left(h_{1}(x)\right)=h_{1}\left(\frac{m-1}{m+1}(a+c)\right) \\
& =-\left(\frac{m-1}{m+1}\right)^{m-1}(a+c)^{m} .
\end{aligned}
$$

Combining this with (11) and (12), we arrive at the following result:

$$
\left(\frac{m-1}{m+1}\right)^{m-1}(a+c)^{m} \leq b \Rightarrow h_{1}(x) \geq-b \Rightarrow h^{\prime}(x) \geq 0 .
$$

Hence the following result has been established.

Proposition 2. Let $f$ be given as a function in the form (9). Then the following holds:

(i) If $m=1$, then for any positive constants $a, b$ and $c$, the function $f$ has a unique fixed point. (ii) If $m=2,3, \ldots$ and the positive constants $a, b$ and $c$ satisfy

$$
\left(\frac{m-1}{m+1}\right)^{m-1}(a+c)^{m} \leq b
$$

then $f$ has a unique fixed point.

Proof. We already proved the case (ii). For the case where $m=1$, let $a, b$ and $c$ be arbitrary positive constants. If $y$ is a fixed point of the function $f$, we have

$$
h(y)=y^{2}+(b-a-c) y-b c=0 .
$$

But $h$ can have at most two roots. Since

$$
h(0)<0 \quad h(-\infty)=\infty,
$$

$h$ has only one positive root; so, $f$ has a unique fixed point.

We have said in Theorem 1 that under positive feedback, the solution converges to one of the equilibrium points independent of delay, see also Smith (2008). Therefore, there should always exist at least one equilibrium point which is locally stable. The following result establishes this property:

Proposition 3. Consider the system (2) under positive feedback, i.e., $g$ defined in (5) satisfies:

$$
g^{\prime}(x)>0 \quad \forall x \in \mathbb{R}_{+} .
$$

Suppose that $g$ is bounded and continuously differentiable, then $g$ has a fixed point $x_{1} \in \mathbb{R}_{+}$such that

$$
g^{\prime}\left(x_{1}\right) \leq 1 \text {. }
$$

Thus, the system is locally stable around the equilibrium point $x_{e q}=\left(x_{1}, x_{2}, \ldots, x_{n}\right)$, where

$$
x_{n}=g_{n}\left(x_{1}\right) / \lambda_{n}, \ldots, x_{2}=g_{2}\left(x_{3}\right) / \lambda_{2} .
$$

Proof. Since the function $g$ is bounded, the following supremum is well-defined:

$$
a=\sup _{x \in \mathbb{R}_{+}}(g(x)) .
$$

It is clear that if $x$ is a fixed point of $g$, then $x \leq a$. Let the set $S$ be defined as

$$
S=\left\{x \in \mathbb{R}_{+}: g(x)=x\right\},
$$

then, because of (13), $b=\sup (S)$ exists. Note that since $g$ is bounded and positive, the set $S$ is nonempty. Since $b=\sup (S)$, there exists a sequence $x_{i} \in S$ such that

$$
g\left(x_{i}\right)=x_{i} \text { and } \lim _{i \rightarrow \infty}\left(x_{i}\right)=b .
$$

Since $g$ is continuous, we have

$$
g(b)=b \text {. }
$$

Suppose that for all fixed points $x$ of $g$, we have

$$
g^{\prime}(x)>1 \text {. }
$$

Then, $g(b)=b$ and $g^{\prime}(b)>1$, but since $g$ bounded then $\exists z>b$ such that

$$
g(z)=z
$$

But this is contradiction to (13), so there exists some $x_{1} \in \mathbb{R}_{+}$such that

$$
g^{\prime}\left(x_{1}\right) \leq 1
$$

The system has the following linearized transfer function around the equilibrium point $x_{e q}$ :

$$
G(s)=\left(1+\frac{g^{\prime}\left(x_{1}\right) \prod_{i=1}^{n}\left(\lambda_{i}\right) e^{-\tau s}}{\prod_{i=1}^{n}\left(s+\lambda_{i}\right)}\right)^{-1} .
$$


The system is locally stable around $x_{e q}$ if the roots of $G(s)$ are in the left half plane. Combining (14) and the fact that the system is under positive feedback, we can verify the following:

$$
0 \leq g^{\prime}\left(x_{1}\right) \leq 1
$$

By applying small gain argument, we can see that the system is locally stable independent of the delay value $\tau$.

\section{EXAMPLES}

We now illustrate the theoretical results obtained in the previous section by examples.

Example 1. Let the function $f$ be in the following form:

$$
f(x)=\frac{3.6 x^{2}}{5+x^{2}}+0.4 .
$$

Let $n=3$, in this case the system has two equilibrium points

$$
e_{1}=(1,1,1), \quad e_{2}=(2,2,2) .
$$

From Theorem 1, we expect the general solution of the system either to converge to $e_{1}$ or to $e_{2}$. First, we present the simulation result shown in Figure 1 which corresponds to initial conditions $x_{1}(0)=0.9, x_{2}(0)=0.95$ and $x_{3}(0)=0.85$ and time delay $\tau=0$ (here, we give the result corresponding to $x_{1}(t)$, other coordinates show similar behavior). As can be seen from Figure 1, the solution converges to the equilibrium point $e_{1}$. Next, we simulate the same system with initial conditions $x_{1}(0)=1, x_{2}(0)=$ $3, x_{3}(0)=4$ and time delay $\tau=2$. The simulation results is shown in Figure 2. When we change the initial conditions, the system converges to the other equilibrium $e_{2}$ which is compatible with the theoretical results we obtained.

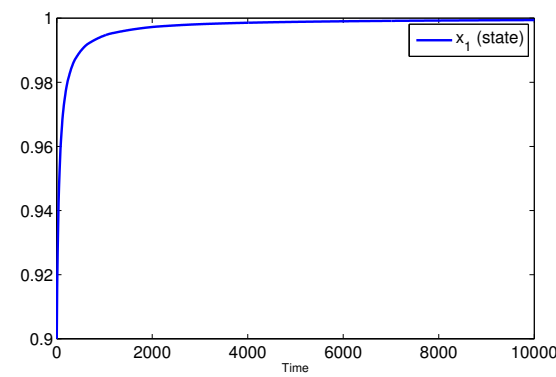

Fig. 1. $x_{1}(t)$ vs $t$ graph for the homogenous gene regulatory network under positive feedback with $\tau=0$.

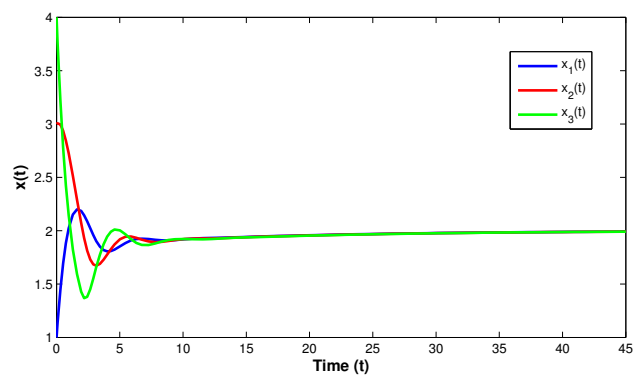

Fig. 2. $x_{1}(t), x_{2}(t), x_{3}(t)$ and $\tau=2$ vs $t$ graph with $x(0)=(0.9,0.95,0.85)$.
Example 2. In this example we will investigate the positive feedback with $n=3$ and having the following nonlinearity function

$$
f(x)=\frac{2 x}{2+x}+1 .
$$

This gives the unique equilibrium point $x_{e q}=(2,2,2)$, so we expect the solutions to converge to $x_{e q}$ for any arbitrary initial condition and time delay. Figures 3 and 4 show the simulation results of the system corresponding to the initial conditions $x(0)=(3,0.5,4), \tau=0$ and $x(0)=(5,3,0.7), \tau=5$ respectively. As we expect the solution converges to the unique equilibrium point $x_{e q}$.

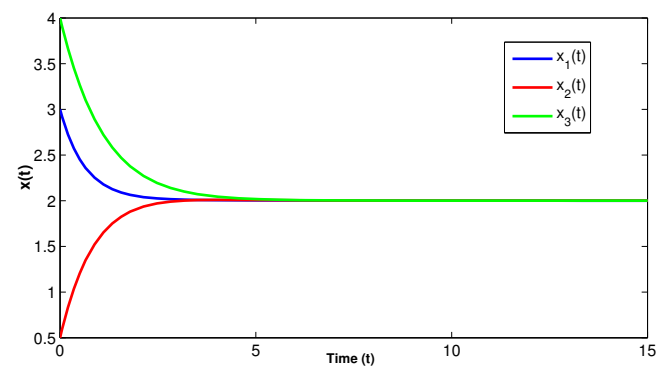

Fig. 3. $x_{1}(t), x_{2}(t)$ and $x_{3}(t)$ vs $t$ graph with $x(0)=$ $(3,0.5,4), \tau=0$.

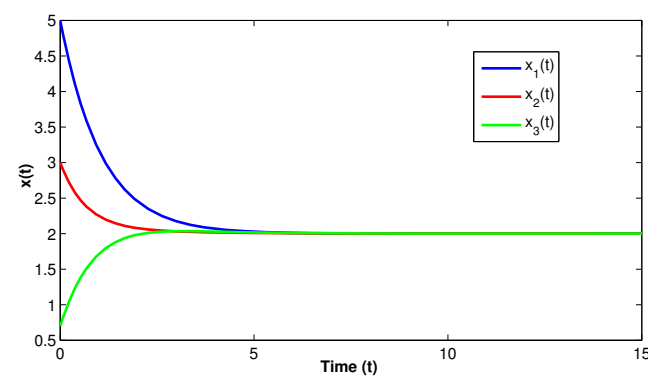

Fig. 4. $x_{1}(t), x_{2}(t)$ and $x_{3}(t)$ vs $t$ graph with $x(0)=$ $(5,3,0.7), \tau=5$.

Example 3. In this example, we will investigate the homogenous positive feedback case with three equilibrium points. Namely, consider the system (2) with $\lambda_{i}=1$ and $g_{i}(x)=f(x)$ is given by

$$
f(x)=g \circ g(x),
$$

where $g$ has the following form:

$$
g(x)=\frac{2}{0.25+x^{3}} .
$$

The function $g$ has the unique fixed point $y_{2}=1.1442$ and the function $f$ has $y_{1}=0.0039, y_{2}=1.1442$ and $y_{3}=8$ as its three fixed points. Therefore, the system has three equilibrium points $z_{1}=\left(y_{1}, y_{1}, y_{1}\right), z_{2}=\left(y_{2}, y_{2}, y_{2}\right)$ and $z_{3}=\left(y_{3}, y_{3}, y_{3}\right)$. If we calculate the derivative of $f$ at its fixed points, we get the following results:

$$
f^{\prime}\left(y_{1}\right)=f^{\prime}\left(y_{3}\right)=2.13 \cdot 10^{-6}<1 \quad f^{\prime}\left(y_{2}\right)=6.6>1 .
$$

The characteristic equation $H_{\tau}^{i}(s)$ of the linearized system around each $z_{i}$ is given by the following formula:

$$
H_{\tau}^{i}(s)=1+\frac{\left(f\left(y_{i}\right)\right)^{3} e^{-\tau s}}{(s+1)^{3}} \quad i=1,2,3 .
$$

Since we have

$$
\left(f\left(y_{i}\right)\right)^{3}<1 \quad \text { for } \quad i=1,3,
$$


the system is locally stable independent of delay around $z_{1}$ and $z_{3}$. The linearized system around $z_{2}$ has the following characteristic equation:

$$
H_{\tau}^{2}(s)=1+G_{\tau}(s)=1+\frac{288 e^{-\tau s}}{(s+1)^{3}} .
$$

It can be shown that the system is unstable independent of delay in this case. Therefore, we expect the solution to converge to either $z_{1}$ or $z_{3}$. Figure 5 shows the solution of the system with $x(0)=(1,1.2,1.4), \tau=0$. Although $x(0)$ is near to $z_{2}$ the solution converges to $z_{3}$. Figure 6 shows the simulation results of the system with $x(0)=$ $(1,0.9,0.8)$ and $\tau=2$. Again, $x(0)$ is near to $z_{2}$ but the solution converges to $z_{1}$ which confirms our theoretical expectations.

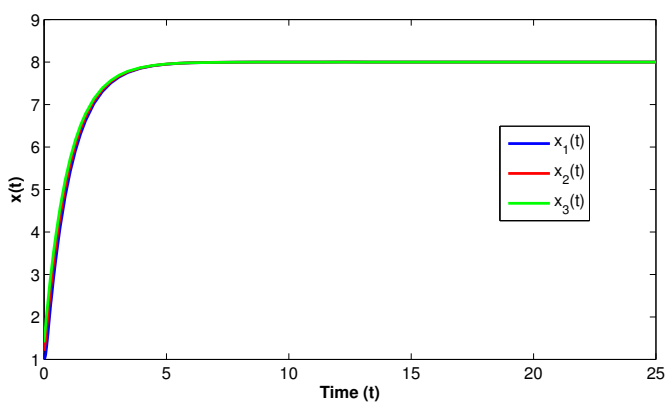

Fig. 5. $x_{1}(t), x_{2}(t)$ and $x_{3}(t)$ vs $t$ graph with $x(0)=$ $(1,1.2,1.4), \tau=0$

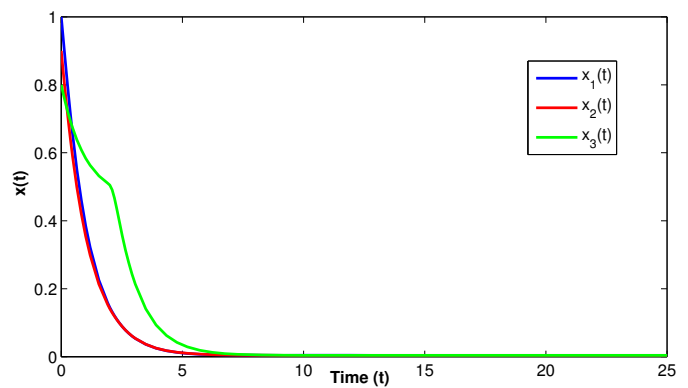

Fig. 6. $x_{1}(t), x_{2}(t)$ and $x_{3}(t)$ vs $t$ graph with $x(0)=$ $(1,1.2,1.4), \tau=0$

\section{CONCLUSIONS}

In this work we considered gene regulatory networks modeled as cyclic nonlinear dynamical systems with time delayed feedback. We analyzed the gene regulatory network under positive feedback and under the assumption that the nonlinearity functions have negative Schwarzian derivatives.

We derived conditions for single positive equilibrium point, which is asymptotically stable independent of delay. In some cases there are more than one equilibrium point. For these situations, we demonstrated how to compute these equilibrium points and whether they are stable or not. We also investigated the homogenous network under positive feedback and derived sufficient conditions for asymptotic stability. As a special case, homogenous gene regulatory networks with Hill type of nonlinearities are considered and sufficient conditions depending only on the parameters of the nonlinearity, are derived for the asymptotic stability independent of delay.

In this paper, we have shown that multiple stable equilibrium points may exists for the system (2). An interesting question as a future extension of the current work is to estimate the radius of convergence for each stable equilibrium point.

\section{REFERENCES}

Ahsen, M.E. (2011). Analysis of two types of cyclic biological system models with time delays. MS Thesis, Graduate School of Engineering and Sciences, Bilkent University, Ankara, Turkey, July 2011.

Morarescu, C. I., Niculescu, S-I. (2008). Some remarks on the delay effects on the stability of biochemical networks. 16th Mediterranean Conference on Control and Automation, 801-805.

Angeli, D., Sontag, E. D. (2004). Multistability in monotone input/output systems. Systems Control Letters, 51, $185-202$.

Levine, M., Davidson, E. H. (2005). Gene regulatory networks for development. Proceedings of the National Academy of Sciences, 102, no. 14, 4936-4942.

Liz, E., Pinto, M., Robledo, G., Trofimchuk, S., Tkachenko, V. (2003). Wright type delay differential equations with negative Schwarzian. Discrete and Continuous Dynamical Systems, 9, No. 2, 309-321.

Enciso, G.A. (2006). On the asymptotic behaviour of a cylic biochemical system with delay. Proceedings of the 45th IEEE Conference on Decision and Control, 23882393.

Müller, S., Hofbauer, J., Endler, L., Flamm, C., Widder, S., Schuster, P. (2006). A generalized model of the repressilator. Journal of Mathematical Biology, 53, 905937.

Goldbeter, A. (1996). Biochemical Oscillations and Cellular Rythms. The Molecular Basis of Periodic and Chaotic Behavior. Cambridge University Press.

Chen, L., Aihara, K. (2002). Stability of genetic regulatory networks with time delay. IEEE Transactions on Circuits and Systems I: Fundamental Theory and Applications, 49, No. 5, 602-608.

Scheper, T. o., Klinkenberg, D., Pennartz, C., Pelt, J. v. (1999). A mathematical model for the intracellular circadian rhythm generator. The Journal of Neuroscience, $19,40-47$.

Sedeghat, H. (2003). Nonlinear Difference Equations. Kluwer Academic Publishers.

Smith, H. (2008). Monotone Dynamical Systems: An introduction to the theory of competitive and cooperative systems. American Mathematical Society.

Smolen, P., Baxter, D.A. and Byrne, J.H. (2000a). Modeling transcriptional control in gene networks - Methods, recent results and future directions Bull. Math. Biol., 62, 247-292.

Smolen, P., Baxter, D.A. and Byrne, J.H. (2000b). Mathematical modeling of gene networks Neuron, 26, 567-580.

Sontag, E. D. (2002). Asymptotic amplitudes and Cauchy gains: a small-gain principle and an application to inhibitory biological feedback. Systems Control Letters, 47, 167-179. 